\title{
Cualidades gerenciales y su influencia en el desempeño empresarial
}

\section{Managerial qualities and their influence on business performance}

Gabriela Duque Espinoza

Universidad del Azuay, Cuenca, Ecuador

gduque@uazuay.edu.ec

iD https://orcid.org/0000-0003-3783-1791

Fernando Córdova León

Universidad del Azuay, Cuenca, Ecuador

jfcordova@uazuay.edu.ec

iD https://orcid.org/0000-0002-7511-688X

Karla González Soto

Universidad del Azuay, Cuenca, Ecuador

kngonzalez@uazuay.edu.ec

(D) https://orcid.org/0000-0001-9725-880

Juan Carlos Aguirre Quezada

Universidad del Azuay, Cuenca, Ecuador

jcaguirre@uazuay.edu.ec

(D) https://orcid.org/0000-0002-8953-8151

Recepción: 01/08/2021 | Aceptación: 01/09/2021 | Publicación: 10/09/2021

Cómo citar (APA, séptima edición):

Duque Espinoza, G., Córdova León, F., González Soto, K., y Aguirre Quezada, J. C. (2021). Cualidades gerenciales y su influencia en el desempeño empresarial. INNOVA Research Journal, 6(3), 155-170. https://doi.org/10.33890/innova.v6.n3.2021.1873

\section{Resumen}

El desempeño empresarial está determinado por factores estructurales que caracterizan al entorno en el que la organización se desenvuelve, sobre los cuales las firmas no pueden incidir de manera directa, y factores endógenos que responden a la gestión estratégica de la empresa; por lo tanto, sus resultados son explicados por las acciones de la gerencia y sus capacidades. El presente trabajo tiene como objetivo determinar la influencia de las cualidades gerenciales sobre el desempeño 
empresarial, para lo cual se analizan las empresas pertenecientes a cuatro sectores manufactureros representativos del Ecuador. Se estima un modelo de regresión que explica el desempeño empresarial mediante variables como el género, formación académica, afinidad de la formación y tiempo de permanencia en el cargo de la gerencia. Los resultados indican que el género femenino, la afinidad de la formación académica y la permanencia en el cargo se relacionan positivamente con el desempeño empresarial.

Palabras claves: cualidades gerenciales; género; formación académica; desempeño empresarial.

\begin{abstract}
Business performance is determined by structural factors that characterize the environment in which the organization operates, on which firms cannot directly influence, and endogenous factors that respond to the strategic management of the company; therefore, its results are explained by the actions of the management and its capacities. The present work aims to determine the influence of managerial qualities on business performance, for which the companies belonging to four representative manufacturing sectors of Ecuador are analyzed. A regression model is estimated that explains business performance through variables such as gender, academic training, affinity of training and time spent in the position of management. The results indicate that the female gender, the affinity of the academic training and the permanence in the position are positively related to the business performance.
\end{abstract}

Keywords: CEO qualities; gender; academic degree; business performance.

\title{
Introducción
}

El entorno en el que se realizan los negocios y los factores organizacionales que se relacionan con la gestión influyen en el desempeño empresarial (Nunes y Pereira, 2021). Las firmas no pueden modificar el medio en el cual se desenvuelven; por lo que, sus resultados se presentan como reflejo de las estrategias implementadas por sus administradores (Saavedra, 2018).

Bajo esta premisa, el liderazgo y el estilo de gestión, definidos por la gerencia, son determinantes para el éxito empresarial (Hoang et al., 2020), se destacan aspectos de índole cualitativa, inherentes a la persona que administra la firma, tales como: experiencia, formación profesional, liderazgo y creatividad, factores que determinan la adquisición y desarrollo del capital intelectual de la organización, ambiente laboral, motivación y productividad (Amelia et al., 2021).

Las organizaciones requieren determinadas capacidades y cualidades por parte de sus administradores en búsqueda de la competitividad y sostenibilidad empresarial (Ramos 2003); dentro de las cuales, resalta la profesionalización como el pilar fundamental en el crecimiento y consolidación de las compañías (Rueda, 2011). Como consecuencia, se establecen estándares de cualidades directivas o condiciones que las empresas desean encontrar en sus futuros directivos, un conjunto de las capacidades estratégicas y de desarrollo de las personas que guían la organización (García et al., 2001).

Esta investigación tiene por objeto determinar la influencia de las cualidades gerenciales sobre el desempeño empresarial; para lo cual, se consideran cuatro sectores representativos de la actividad manufacturera del Ecuador: cárnicos, bebidas, muebles y textiles. Se utiliza información 
secundaria correspondiente a los estados financieros que las empresas catalogadas como sociedades reportan anualmente a la Superintendencia de Compañías Valores y Seguros del Ecuador (2021). Además, se recopiló información respecto al género, formación académica y permanencia en el cargo de los gerentes de las firmas de estos sectores para el año 2018. Se estima para cada sector económico estudiado un modelo de regresión lineal múltiple que permite evidenciar la influencia de las cualidades gerenciales sobre el desempeño empresarial.

Los resultados indican una proporción minoritaria de gerentes de género femenino en los sectores de estudio, situación que refleja la escasa participación de mujeres en las actividades empresariales en Ecuador. Además, la mayor proporción de gerentes cuentan con formación académica de bachillerato y se evidencia que la titulación de cuarto nivel en la administración es reducida. Se destaca la influencia positiva de la gerencia femenina y la afinidad de la formación académica en el desempeño empresarial, hallazgo que se replica en todos los sectores de estudio.

El estudio en su inicio expone el marco teórico y el estado del arte de la temática abordada; luego, se detalla la metodología aplicada, la composición de la unidad de análisis y la especificación del modelo de regresión; a continuación, se presentan los resultados obtenidos y se discuten los hallazgos más relevantes; para finalmente, dará conocer las conclusiones y recomendaciones derivadas de la investigación.

\section{Estado del arte y marco teórico}

El desempeño empresarial está atado simultáneamente a factores como el capital, el talento humano, la innovación, el proceso productivo, entre otros (Flores-Romero y González-Santoyo, 2019); sin embargo, uno de los más importantes es la administración, mediante la toma de decisiones asertiva enfocada en la creación de valor (Firk et al., 2021).

Una empresa se enfrenta a elementos estructurales que conforman el entorno económico y legal, sobre los cuales no puede incidir directamente; por lo tanto, el éxito de sus actividades responde a la efectividad de la estrategia y tácticas que se implementen con la finalidad de adaptarse al medio y aprovechar las oportunidades que presenta (Hoang et al., 2020). En este proceso, es fundamental, el papel que la gerencia lleva a cabo para diseñar e implementar la estrategia organizacional; por lo tanto, se puede inferir que el desempeño empresarial se relaciona con las capacidades y fortalezas de la administración (Firk et al., 2021; Odiri y Ideh, 2021).

La directiva de una compañía, encabezada por el gerente, cumple el rol de líder y guía de la organización, señala el camino y los objetivos a cumplir por parte de la empresa (Chen et al., 2021). De igual manera, desempeña el papel de máxima autoridad en cuanto al control y evaluación de la firma; pues, la gerencia tiene la tarea de cerciorarse del correcto desenvolvimiento de las labores en la organización de acuerdo con su planificación estratégica (Ramírez et al., 2018).

El estilo de liderazgo repercute en la administración de distintas maneras; puesto que, las cualidades de un gerente determinan su estilo de gestión. La literatura define a los líderes estratégicos como aquellos que enfatizan y resaltan la importancia de las contribuciones individuales en todos los niveles de la empresa y fomentan nuevas formas de abordar situaciones existentes (Cortes y Herrmann, 2021), lo que da lugar a un contexto laboral que estimula la 
creatividad (Oorschot et al., 2021). Este tipo de liderazgo se relaciona con la motivación, productividad y retención del talento (Krén y Séllei, 2020; McCormick et al., 2021); además, con la resolución de conflictos en equipos multigeneracionales, (Bhayana et al., 2021) lo que propicia la innovación disruptiva y el comportamiento intraemprendedor (Cortes y Herrmann, 2021; Gahan et al., 2021; Mishra, 2021; Usman et al., 2021). El estilo de gerencia basado en el liderazgo estratégico genera confianza en los directores de las áreas de la organización, lo cual tiene un impacto positivo en el desempeño de las pymes (Susanto et al., 2021).

La competitividad de una empresa se presenta como un reflejo de su administración (Susanto et al., 2021); por esta razón, las organizaciones requieren determinadas capacidades y cualidades por parte de sus administradores en búsqueda del desarrollo y sostenibilidad empresarial (Ramos, 2003). Dentro de estas, resalta la profesionalización como el pilar fundamental en el crecimiento y consolidación de las compañías (Rueda, 2011). Es por esto que las empresas determinan perfiles personales y profesionales que reúnen las cualidades deseadas para estar en la administración (García et al., 2001).

Estos estándares responden a aspectos cualitativos, que permiten a las organizaciones identificar el estilo de liderazgo que desean implementar, con miras a consolidar una posición competitiva y el cumplimiento de objetivos de su planificación estratégica; por lo tanto, es preciso estudiar la manera en la que estas cualidades repercuten sobre el desempeño empresarial.

Varias investigaciones han buscado observar diferencias en el desempeño empresarial en función del género del administrador o gerente. Omar y Pérez (2014) concluyen que existe una relación positiva y significativa entre el género femenino y las ganancias de la empresa, y fundamentan este hallazgo en el estilo de gestión y la profesionalización de las gerentes, aspectos que tienen repercusión sobre la productividad y motivación de los colaboradores.

Saavedra (2018) afirma que la competitividad de las empresas dirigidas por mujeres es menor tanto por las restricciones sociales existentes y por la dificultad para acceder a recursos externos; concluye que, no existe diferencia en el nivel de ventas entre la administración de hombres y mujeres, los activos de las empresas dirigidas por mujeres son menores a las dirigidas por hombres y que el capital es mayor para las empresas dirigidas por hombres; sin embargo, no encuentran evidencia de una diferencia significativa del desempeño de las firmas en función del género de la gerencia.

Diéguez et al. (2010) analizan las diferencias en el éxito empresarial en función del género de la gerencia; a través, de variables como el número de empleados, el crecimiento de la empresa, niveles de endeudamiento, la evolución de la situación financiera y la satisfacción de los clientes. La investigación evidencia que las mujeres tienen una percepción más precisa de los resultados y sus éxitos que los hombres, y que las empresas dirigidas por mujeres tienen mayor acceso a recursos financieros; por lo que, sus niveles de endeudamiento son superiores.

García et al. (2017) estudian el comportamiento de las PYMES con base en el género de la gerencia; encuentran que la edad de la empresa no es relevante con relación al género de la gerencia; además, evidencian que, en sectores de alta tecnología, como los manufactureros, predomina el género masculino en la administración; y se observa una tendencia en la gerencia 
masculina a reducir empleados, situación contraria en la administración femenina que tiende a aumentarlos.

Además del género, otra característica de interés en la gerencia es la formación académica. García et al. (2017) al hablar del nivel de formación de los administradores observan que cerca del $80 \%$ de los gerentes hombres tienen formación universitaria a diferencia de las Pymes gestionadas por mujeres que poseen más gerentes con estudios básicos. Aragón et al. (2003) exponen indicios de una relación positiva entre la formación académica de los directivos y los resultados empresariales reflejados en la rentabilidad y eficacia, especialmente al momento de dar el salto desde la economía sumergida hacia la formalidad.

Rueda (2011) manifiesta que la profesionalización es fundamental en el crecimiento y consolidación de las compañías, en especial de las de menor tamaño; por tanto, la formación y capacitación de sus directivos cobra una gran significancia. En este sentido, García et al. (2001) consideran que la instrucción formal aporta al desarrollo de capacidades y destrezas que las empresas desean encontrar en sus futuros directivos y que favorecen la administración enfocada en la creación de valor; en adición, Li (2017) sostiene que la formación afín a la administración empresarial potencia el desempeño de los gerentes al momento de alcanzar sus objetivos. En concordancia, Lalic et al. (2019) enfatizan la importancia de la formación académica, en especial, en emprendedores o empresarios que carecen de instrucción básica; debido a que, identifican una relación positiva entre el grado universitario y las capacidades de innovación y liderazgo.

No obstante, existen autores que sostienen que la formación de los estudiantes universitarios no corresponde con las exigencias requeridas por las compañías en el sector laboral. Por ejemplo, Delgado et al. (2019) encuentran que únicamente el 58\% de las competencias y conocimientos aplicados en las empresas son adquiridas durante la etapa de grado de estudios. Martínez (2009) manifiesta que los conocimientos base proporcionados por la universidad son adecuados pero se incita a esta institución a redirigir su enfoque para abarcar también el desarrollo de aptitudes transversales como el trabajo en equipo, solución de problemas y toma de decisiones.

Otra cualidad relevante al momento de administrar una firma es la experiencia, entendida como el tiempo ininterrumpido de ejercicio en el cargo. Tu et al. (2020) muestran que el desempeño de las empresas se fortalece cuando el gerente tiene más años de experiencia dirigiendo la misma organización. La experiencia es un indicador que refleja las habilidades y competencias cognitivas de la gerencia, la cual tiene una influencia positiva significativa en el nivel de competitividad en mercados locales e internacionales (Bany-Ariffin et al., 2014).

El estilo de liderazgo ejercido por un administrador con experiencia puede llegar a estimular la innovación en una empresa y potenciar el crecimiento estratégico mediante la internacionalización de operaciones; por lo que, los propietarios de una firma, brindan especial atención a la experiencia de los candidatos a dirigir la organización (Li, 2017). León (2018) manifiesta que existe una relación directa y significativa entre las variables de tiempo de permanencia en el cargo de directores y la calidad de gestión administrativa; debido a que, los gerentes más experimentados presentan mejores aptitudes en el proceso de toma de decisiones; además de, despertar mayor confianza en inversionistas y acreedores, aspectos que facilitan la obtención de recursos en condiciones favorables. 
Del análisis precedente se desprende la importancia que tienen las cualidades inherentes a la persona que administra una firma; puesto que, estas pueden influenciar la manera en la que la empresa interactúa con el medio y gestiona sus recursos; por tanto, es preciso determinar la forma en la que las cualidades de la gerencia repercuten sobre el desempeño empresarial. El presente trabajo aporta a la literatura con evidencia empírica que describe la influencia del género, formación académica y experiencia de la gerencia, sobre el desempeño económico de las organizaciones, en una economía emergente con alta incidencia de la informalidad (CaminoMogro y Brito-Gaona, 2021; Montiel, 2021; Varela, 2020), con la finalidad de evidenciar las cualidades deseadas en la administración con miras a potencializar el desempeño empresarial.

\section{Metodología}

La presente investigación es de tipo descriptivo, y busca determinar la influencia de las cualidades gerenciales sobre el desempeño empresarial, mediante el estudio de cuatro sectores representativos de la actividad manufacturera del Ecuador: cárnicos, bebidas, muebles y textiles. Se utiliza información secundaria correspondiente a los estados financieros que las empresas catalogadas como sociedades reportan a la Superintendencia de Compañías Valores y Seguros del Ecuador (2021). Además, se recopiló información respecto al género, formación académica y permanencia en el cargo de los gerentes de las firmas de estos sectores para el año 2018.

\section{Tabla 1}

Priorización de sectores a estudiar, año 2018

\begin{tabular}{lccccc}
\hline Sector económico & $\begin{array}{l}\text { Número de } \\
\text { empresas }\end{array}$ & Empleados & Activos & Ventas & Utilidad \\
\hline Cárnico & 99 & 16.496 & $1.089,53$ & 566,42 & $1.604,68$ \\
Bebidas & 197 & 11.160 & $1.281,98$ & 402,38 & $1.654,67$ \\
Textil & 318 & 9.167 & 343,60 & 150,48 & 379,73 \\
Muebles & 164 & 4.048 & 191,28 & 79,17 & 191,85 \\
\hline Total & 778 & 40.871 & $2.906,38$ & $1.198,44$ & $3.830,93$ \\
\hline
\end{tabular}

Nota: Activos, ventas y utilidad expresados en millones de dólares.

La tabla 1 presenta la composición de la unidad de análisis, la cual comprende 778 empresas correspondientes a los sectores de estudio; además, se analizan de forma comparativa las distintas cualidades de la gerencia de las firmas con la finalidad de conocer la composición de la administración y la influencia de sus cualidades sobre el desempeño empresarial.

Se estima para cada sector económico estudiado un modelo de regresión lineal múltiple que permite evidenciar la influencia de las cualidades gerenciales sobre el desempeño empresarial. Se considera como variable dependiente el desempeño empresarial (DES), medido a través del margen de utilidad neta; explicado por el género (GEN), la formación académica (FOR), la afinidad de la formación académica respecto a la administración (AFI) y el tiempo de permanencia ininterrumpida en el cargo por parte de la gerencia (PER); además, se contemplan variables de 
control relacionadas con la gestión financiera como liquidez corriente (LIQ), período promedio de inventario (INV), período promedio de cobo (COB) e índice de endeudamiento (END).

$$
\begin{aligned}
& D E S=\beta_{0}+\beta_{1} G E N+\beta_{2} F O R+\beta_{3} A F I+\beta_{4} P E R+\beta_{5} L I Q+\beta_{6} I N V+\beta_{7} C O B+ \\
& \beta_{8} E N D+\varepsilon_{i}
\end{aligned}
$$

Las variables cualitativas se categorizaron de la siguiente manera: la variable género toma valores de 1 para gerentes femeninos, y de cero para gerentes masculinos; la formación académica toma valores según el grado de formación académica más alto alcanzado por el gerente, esto es 1 para bachillerato, 2 tercer nivel y 3 para cuarto nivel; la variable afinidad se determina como 1 cuando la formación académica del gerente es afín a la administración o carreras semejantes, caso contrario su valor es 0; la permanencia en el cargo se cuantifica como los años de ejercicio del cargo de administrador o gerente de forma ininterrumpida en la misma organización.

Las variables de control se calcularon conforme a la literatura sobre gestión financiera: liquidez corriente (LIQ) como la relación entre activo corriente y pasivo corriente; período promedio de inventario (INV) como el cociente entre 365 días del año y la rotación de inventarios; el período promedio de cobro (COB) como la razón entre 365 días del año y la rotación de cuentas por cobrar; y el índice de endeudamiento (END) como la división entre los pasivos totales y los activos totales.

De esta forma, se estima la influencia que cada variable independiente ejerce sobre el desempeño empresarial con la finalidad de proporcionar a los sectores industriales herramientas que faciliten la toma de decisiones; además, de brindar a los directivos detalles sobre las cualidades o aspectos que el tejido empresarial ecuatoriano valora y busca en sus administradores.

\section{Resultados y discusión}

A continuación, se presentan los hallazgos encontrados, los cuales describen la conformación de la administración de los sectores económicos estudiados, en cuanto al género, formación académica, afinidad de la formación respecto a la administración y permanencia ininterrumpida en el cargo.

\section{Tabla 2}

Estadísticos descriptivos

\begin{tabular}{lrrrrrr}
\hline Variable & Media & Mediana & $\begin{array}{c}\text { Desv. } \\
\text { Estándar }\end{array}$ & p25 & p50 & p75 \\
\hline Muebles & & & & & & \\
DES & $-0,006$ & 0,007 & 0,112 & $-0,011$ & 0,007 & 0,030 \\
PER & 6,528 & 5,314 & 5,388 & 2,568 & 5,314 & 8,408
\end{tabular}




\begin{tabular}{lrrrrrr}
\hline Variable & Media & Mediana & $\begin{array}{c}\text { Desv. } \\
\text { Estándar }\end{array}$ & \multicolumn{1}{c}{ p25 } & p50 & p75 \\
\hline LIQ & 1,838 & 1,260 & 1,363 & 0,892 & 1,260 & 2,607 \\
INV & 63,397 & 47,664 & 46,404 & 27,765 & 47,664 & 105,621 \\
COB & 57,606 & 49,434 & 40,109 & 25,165 & 49,434 & 77,250 \\
END & 0,687 & 0,728 & 0,328 & 0,461 & 0,728 & 0,918 \\
\hline Bebidas & & & & & & \\
DES & 0,004 & 0,012 & 0,091 & $-0,007$ & 0,012 & 0,035 \\
PER & 5,977 & 3,800 & 5,803 & 1,404 & 3,800 & 7,810 \\
LIQ & 1,365 & 1,154 & 0,967 & 0,735 & 1,154 & 1,908 \\
INV & 33,522 & 21,596 & 34,742 & 9,365 & 21,596 & 47,813 \\
COB & 77,261 & 55,139 & 61,277 & 22,604 & 55,139 & 130,175 \\
END & 0,618 & 0,665 & 0,344 & 0,417 & 0,665 & 0,868 \\
\hline Cárnico & & & & & & \\
DES & $-0,113$ & 0,015 & 0,590 & 0,002 & 0,015 & 0,042 \\
PER & 6,646 & 4,518 & 6,346 & 2,158 & 4,518 & 9,042 \\
LIQ & 3,314 & 1,305 & 9,828 & 0,707 & 1,305 & 2,061 \\
INV & 21,125 & 7,807 & 26,776 & 4,153 & 7,807 & 32,420 \\
COB & 48,882 & 34,208 & 47,394 & 17,196 & 34,208 & 60,677 \\
END & 0,524 & 0,608 & 0,404 & 0,008 & 0,608 & 0,879 \\
\hline Textil & & & & & \\
DES & 0,017 & 0,018 & 0,094 & 0,006 & 0,018 & 0,043 \\
PER & 4,462 & 3,160 & 4,817 & 1,806 & 3,160 & 4,725 \\
LIQ & 1,868 & 1,389 & 1,547 & 1,020 & 1,389 & 1,995 \\
INV & 110,463 & 61,326 & 105,723 & 26,205 & 61,326 & 176,967 \\
COB & 119,985 & 97,066 & 90,236 & 41,702 & 97,066 & 174,552 \\
END & 0,695 & 0,746 & 0,304 & 0,514 & 0,746 & 0,924 \\
\hline
\end{tabular}

La tabla 2 presenta los estadísticos descriptivos de las variables analizadas en los sectores de estudio; se observa que, los sectores textil y bebidas, son los que han obtenido, en promedio, mejor desempeño económico; en adición, en los sectores muebles y bebidas se evidencia que una cuarta parte de las organizaciones obtienen pérdidas. En cuanto a la permanencia en el cargo de la administración, se destacan los sectores muebles y cárnicos, como aquellos en los que los gerentes, en promedio, cuentan con mayor tiempo de gestión; en contraposición, en el sector textil, los gerentes muestran los menores tiempos de ejercicio ininterrumpido, dentro de los sectores analizados. 


\section{Figura 1}

Género de la gerencia

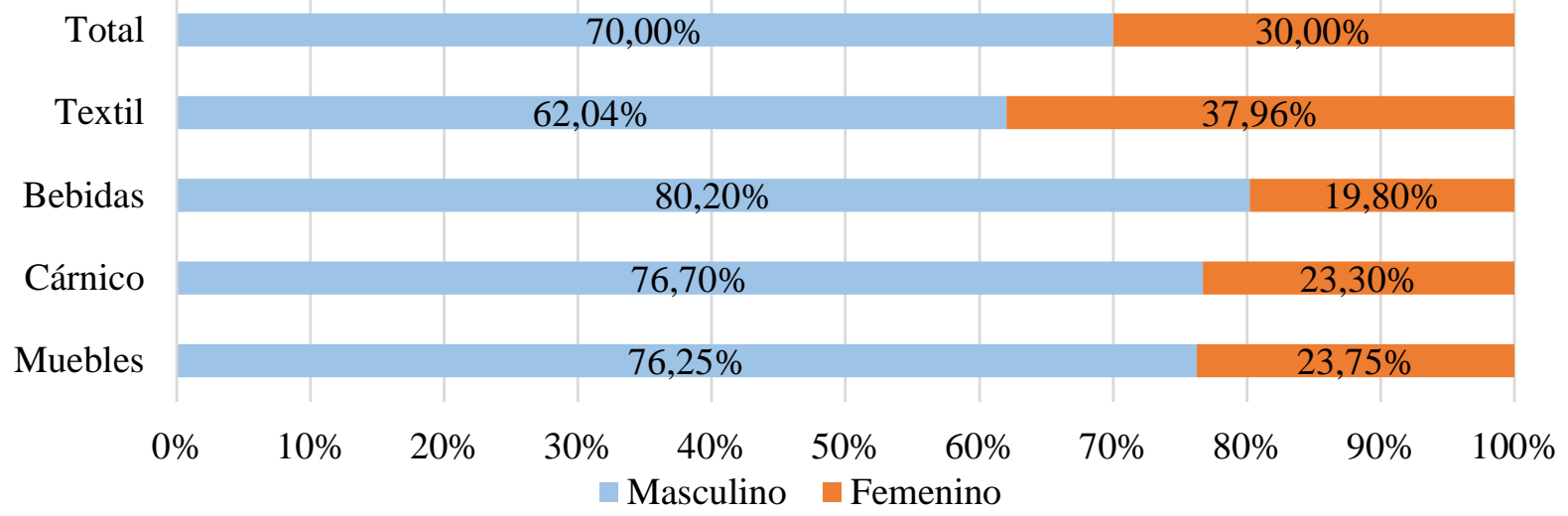

La figura 1 presenta el género de la gerencia en los sectores analizados, se evidencia que la mayor proporción de gestores corresponden al género masculino; por lo tanto, la participación femenina en la administración empresarial queda relegada a una proporción minoritaria. Se destaca al sector textil como aquel en el que la gerencia femenina ha ganado mayor intervención sin llegar a representar la mayor proporción de gerentes. Estos resultados denotan la posible existencia de barreras de índole sociocultural que impiden el acceso de mujeres a cargos directivos, hallazgo que respalda las investigaciones de Diéguez et al. (2010), García et al. (2017) y Saavedra (2018) en donde se subraya la importancia de que las políticas públicas y la cultura organizacional propendan a impulsar la participación femenina en la dirección de las firmas.

\section{Tabla 3}

Formación académica de la gerencia

\begin{tabular}{lrrrrrr}
\hline & \multicolumn{2}{c}{ Bachillerato } & \multicolumn{2}{c}{ Tercer Nivel } & \multicolumn{2}{c}{ Cuarto Nivel } \\
Sector & \multicolumn{1}{c}{ Frecuencia } & Porcentaje & Frecuencia & Porcentaje & Frecuencia & Porcentaje \\
\hline Muebles & 35 & $47,95 \%$ & 31 & $42,47 \%$ & 7 & $9,59 \%$ \\
Bebidas & 34 & $37,78 \%$ & 43 & $47,78 \%$ & 13 & $14,44 \%$ \\
Cárnico & 61 & $59,22 \%$ & 30 & $29,13 \%$ & 12 & $11,65 \%$ \\
Textil & 124 & $61,08 \%$ & 70 & $34,48 \%$ & 9 & $4,43 \%$ \\
\hline Total & 254 & $54,16 \%$ & 174 & $37,10 \%$ & 41 & $8,74 \%$ \\
\hline
\end{tabular}

En la Tabla 3 se observa que el máximo nivel educativo alcanzado por la mayoría de los gerentes dentro de los sectores industriales Textiles, Cárnicos y Muebles es Bachillerato. En el sector Bebidas se evidencia que la mayor parte de gerentes, en específic o el 47,78\% tienen un nivel educativo de Tercer Nivel. La situación es similar cuando se analiza los gerentes con formación de Cuarto Nivel, pues es el sector que mayor porcentaje de administradores con estudios de posgrado presenta; sin embargo, se evidencia una baja proporción de gerentes que buscan mayor profesionalización. 


\section{Tabla 4}

Formación académica y género de la gerencia

\begin{tabular}{lcccccc}
\hline & \multicolumn{2}{c}{ Bachillerato } & \multicolumn{2}{c}{ Tercer Nivel } & \multicolumn{2}{c}{ Cuarto Nivel } \\
Sector & Frecuencia & Porcentaje & Frecuencia & Porcentaje & Frecuencia & Porcentaje \\
\hline Masculino & & & & & & \\
Muebles & 25 & $43,86 \%$ & 25 & $43,86 \%$ & 7 & $12,28 \%$ \\
Bebidas & 23 & $32,39 \%$ & 38 & $53,52 \%$ & 10 & $14,08 \%$ \\
Cárnico & 46 & $58,23 \%$ & 23 & $29,11 \%$ & 10 & $12,66 \%$ \\
Textil & 76 & $61,29 \%$ & 39 & $31,45 \%$ & 9 & $7,26 \%$ \\
\hline Femenino & & & & & & \\
Muebles & 10 & $62.50 \%$ & 6 & $37.50 \%$ & 0 & $0.00 \%$ \\
Bebidas & 11 & $57.89 \%$ & 5 & $26.32 \%$ & 3 & $15.79 \%$ \\
Cárnico & 15 & $62.50 \%$ & 7 & $29.17 \%$ & 2 & $8.33 \%$ \\
Textil & 48 & $60.76 \%$ & 31 & $39.24 \%$ & 0 & $0.00 \%$ \\
\hline
\end{tabular}

En la Tabla 4 se presenta la formación académica con la que cuentan los gestores en función de su género; se observa que, en el caso de gerentes con género femenino, en todos los sectores de estudio, la mayor parte de administradoras cuentan con grado de bachiller; además, se muestra que existe una reducida proporción de gerentes con formación de cuarto nivel. En contraste, los gerentes con género masculino replican esta distribución únicamente en los sectores Cárnico y Textil; en contrapartida, el sector Bebidas, presenta la mayor proporción de gerentes con formación de tercer nivel.

\section{Figura 2}

Género de la gerencia y permanencia en el cargo

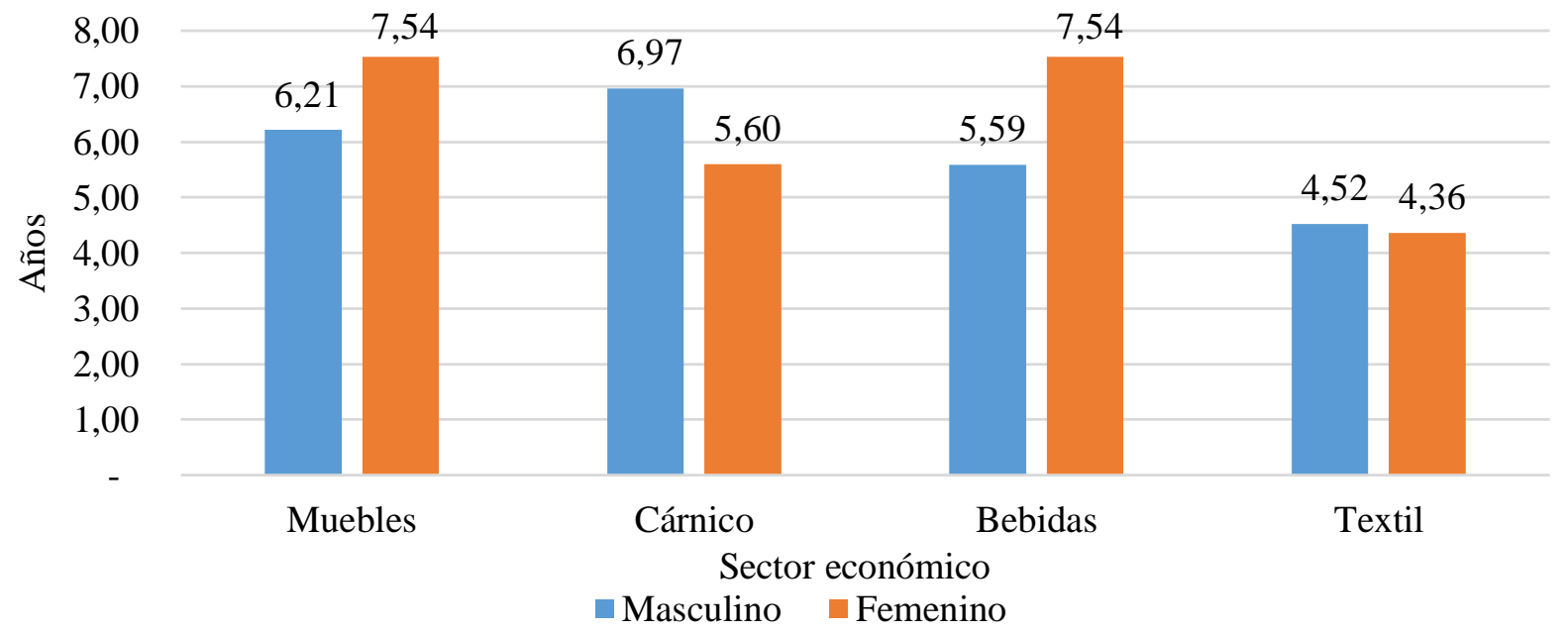

En la figura 2 se puede evidenciar la permanencia ininterrumpida en el cargo de los gerentes de los sectores estudiados en función de su género. Se observa que esta característica depende de la actividad empresarial; se destaca que en los sectores Muebles y Bebidas la permanencia en el 
cargo de gerentes de género femenino es mayor que aquellos de género masculino, caso contrario a lo que ocurre en los otros dos sectores. superior.

\section{Figura 3}

Formación académica de la gerencia y permanencia en el cargo

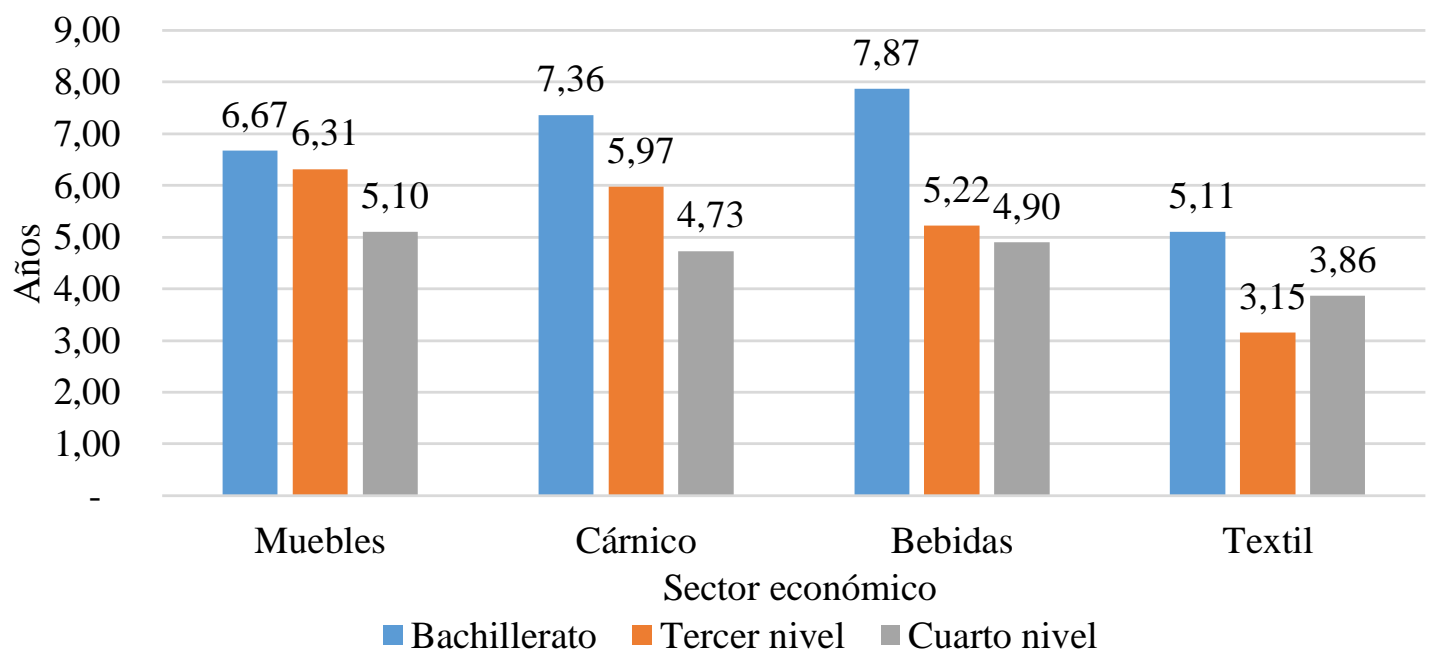

En la figura 3 se expone la formación académica de los gerentes y su tiempo de permanencia en el cargo; se evidencia que, en los cuatro sectores analizados, los gerentes con formación académica de Bachillerato son los que se mantienen por un período más largo en el cargo. Este hallazgo podría ser un indicador de que la rotación de los administradores se incrementa conforme alcanzan mayor formación académica, en búsqueda de alcanzar mejores oportunidades laborales; mientras que, aquellos administradores que cuentan con menor instrucción formal tienen menores incentivos para abandonar su cargo.

\section{Tabla 5}

Estadísticos del modelo de regresión por sector económico

\begin{tabular}{lcccccccc}
\hline \multirow{2}{*}{ Variable } & \multicolumn{2}{c}{ Muebles } & \multicolumn{2}{c}{ Textil } & \multicolumn{2}{c}{ Bebidas } & \multicolumn{2}{c}{ Cárnicos } \\
& $\beta$ & Sig. & $\beta$ & Sig. & $\beta$ & Sig. & B & Sig. \\
\hline Constante & $-0,096$ & 0,353 & 0,227 & 0,136 & $-0,128$ & 0,302 & 0,408 & 0,112 \\
GEN $^{\mathrm{a}}$ & $0,107 * *$ & 0,043 & $0,123 * * *$ & 0,002 & $0,137 * * *$ & 0,010 & $0,068 * * *$ & 0,009 \\
FOR $^{\mathrm{b}}$ & $-0,125$ & 0,456 & $0,021 *$ & 0,061 & $0,006 * *$ & 0,046 & $-0,026$ & 0,665 \\
AFI $^{\mathrm{c}}$ & $0,229 * *$ & 0,031 & $0,035 * *$ & 0,036 & 0,042 & 0,326 & $0,009 *$ & 0,085 \\
PER $^{\mathrm{d}}$ & $0,003 * *$ & 0,048 & 0,000 & 0,905 & $-0,002$ & 0,747 & $0,003 * *$ & 0,050 \\
LIQ $^{*}$ & $0,095 * *$ & 0,011 & $-0,025 * *$ & 0,016 & $0,041 * *$ & 0,035 & $-0,040 * *$ & 0,049 \\
INV & $-0,001 * *$ & 0,043 & $0,001 *$ & 0,080 & $0,093 *$ & 0,082 & 0,000 & 0,800 \\
COB & $-0,003 * *$ & 0,020 & 0,000 & 0,894 & 0,000 & 0,548 & $-0,002 * *$ & 0,014 \\
END & $0,489 * *$ & 0,016 & $-0,326 * * *$ & 0,001 & $0,100 * *$ & 0,028 & $-0,195 * *$ & 0,029 \\
$\mathrm{R}^{2}$ & 0,823 & \multicolumn{2}{c}{0,606} & \multicolumn{2}{c}{0,723} & \multicolumn{2}{c}{0,817} \\
\hline
\end{tabular}

Notas: * estadísticamente significativo al $0,10 * *$ estadísticamente significativo al 0,$05 ; * * *$ estadísticamente significativo al 0,01. 
a. Género de la gerencia: Femenino $=0$, Masculino $=1$.

b. Formación académica de la gerencia: Bachiller $=1$, Tercer Nivel $=2$, Cuarto Nivel $=3$

c. Afinidad de la formación de la gerencia: Afín a la administración o similares $=1$, No afín $=0$

d. Permanencia: Tiempo ininterrumpido de ejercicio de la gerencia en la misma organización en años.

La tabla 5 expone los estadísticos que resumen el modelo de regresión estimado para los sectores de estudio. Se encuentra que, en todos los sectores, el género del gerente influye significativamente sobre el desempeño empresarial; de forma que, el género femenino se relaciona con un mayor margen de utilidad neta. Esta relación concuerda con los resultados de Omar y Pérez (2014) y García et al. (2017) respecto a que la gerencia femenina presenta mejores capacidades analíticas y percepciones más realistas de los resultados, aspectos que derivan en un liderazgo estratégico, que potencia el desempeño empresarial (Cortes y Herrmann, 2021; Gahan et al., 2021; Mishra, 2021; Usman et al., 2021).

En cuanto a la influencia de la formación académica de la gerencia sobre el desempeño empresarial, los sectores estudiados presentan resultados divergentes, lo que puede ser un indicio de que, en algunos sectores industriales el nivel de competitividad y las características propias de la actividad de negocio, demandan administradores con mayor formación académica; además, de las oportunidades de crecimiento profesional para gestores con formación académica de niveles superiores. Los sectores textil y bebidas, muestran que la formación académica de la gerencia influye de manera directa y estadísticamente significativa sobre el desempeño económico de las firmas. Estos hallazgos respaldan lo afirmado por Aragón Sánchez et al. (2003) y Rueda, (2011) respecto a la importancia que tiene la profesionalización del gerente para que el proceso de transición entre la economía sumergida y la economía formal se lleve a cabo con éxito.

La afinidad de la formación académica de la gerencia respecto a la administración presenta una influencia positiva sobre el desempeño empresarial, lo cual ratifica que la formación en áreas relacionadas con la administración proporciona a los gestores herramientas, y desarrolla capacidades y destrezas que les permiten aprovechar óptimamente los recursos productivos con miras a la consecución de objetivos organizacionales (García et al., 2001; Lalic et al., 2019; Li, 2017).

Respecto a la permanencia en el cargo del gerente y su influencia en el desempeño empresarial, se evidencia en los sectores muebles y cárnicos una relación positiva entre estas variables, se destaca el aporte de la experiencia al dirigir la misma organización; puesto que, permite a los administradores desarrollar sus habilidades de adaptación a la organización y disminuir los riesgos al conocer de mejor manera el giro del negocio, lo cual facilita el proceso de toma de decisiones, y genera mayor confianza en los inversionistas y proveedores (León, 2018; $\mathrm{Li}, 2017)$.

Con relación a las variables de control, se evidencia que estas describen adecuadamente los aspectos clave de la gestión financiera de cada sector económico estudiado; puesto que, influyen 
de manera distinta sobre el desempeño empresarial, lo que denota las particularidades que tiene cada sector y en específico su gerencia financiera.

Los resultados demuestran la influencia de ciertas características que debe tener la persona que gerencia una firma para obtener un mayor desempeño en la organización, al encontrar una relación positiva entre la gestión femenina y el margen de utilidad; así como, evidenciar la importancia de la profesionalización de los administradores en carreras afines a la gestión empresarial; cualidades que deben ser consideradas por las organizaciones ecuatorianas, al momento de la selección de gerentes que potencien su crecimiento económico.

\section{Conclusiones y recomendaciones}

La presente investigación analiza los aspectos cualitativos inherentes a la persona que administra una firma y cómo estos repercuten sobre el desempeño empresarial, al identificar rasgos que definen el estilo de gestión y liderazgo ejercido por el administrador.

Los principales hallazgos destacan que, a pesar de que la literatura y los resultados obtenidos sugieren que el género femenino de la gerencia se relaciona con mayor desempeño empresarial, la actividad gerencial recae mayoritariamente sobre los hombres, relegando a una menor proporción la participación femenina; esta situación, denota posibles barreras culturales y sociales que impiden a la mujer ser partícipe en mayor medida de la gerencia y que imposibilitan a la economía en su conjunto beneficiarse de las cualidades femeninas de gestión; puesto que, la gerencia femenina ha demostrado mayor desempeño empresarial.

Es importante destacar la relevancia que tiene la formación académica de los gerentes en el desempeño económico; en especial, la importancia de que los administradores alcancen estándares mínimos de profesionalización, que les permita adquirir herramientas de gestión encaminadas a la formalización y sostenibilidad de las unidades económicas. Respecto a la formación de cuarto nivel, los hallazgos indican que conforme los gerentes alcanzan mayor instrucción formal, se evidencian menores períodos de permanencia en el cargo, aspecto que puede relacionarse con mayores expectativas de desarrollo profesional y personal.

La experiencia de administración en la misma organización se relaciona favorablemente con el desempeño empresarial, este hallazgo resalta la importancia de dotar a los gerentes de un período de tiempo destinado al desarrollo de una curva de aprendizaje sobre los aspectos estratégicos del entorno, sector económico y particularidades de la organización; de esta forma, el administrador obtiene las competencias que la organización demanda conforme a su planificación estratégica.

El presente trabajo recalca la importancia de las cualidades inherentes a la persona que ejerce la administración como determinantes del desempeño empresarial; sin embargo, es preciso mencionar que, estas cualidades interfieren también en la reputación organizacional (Ogunfowora, 2014) y su posicionamiento frente a los clientes internos (Sant, 2016) y externos (Men, 2012); debido a que, el gerente es quien personifica los valores y principios de la firma . Por esta razón, aspectos como el estilo de gestión en función de las cualidades del gerente, las barreras que 
impiden la participación femenina en la gerencia y estrategias para impulsarla, son líneas de investigación futuras que se derivan del presente trabajo.

\section{Referencias bibliográficas}

Amelia, D., Syukmayettil, L., y Zuripal. (2021). The Effect of Business Knowledge, Business Skill, Self Confidence and Innovation on Business Performance of Small and Medium Industry in the City of Bukittinggi. IOP Conference Series: Earth and Environmental Science, 747(1), 012084. https://doi.org/10.1088/1755-1315/747/1/012084

Aragón, A., Sanz, R., y Barba, M. I. (2003). Efectos de la formación de directivos en las Pymes españolas. Estudios financieros. Revista de Trabajo y Seguridad Social: Comentarios, casos prácticos: recursos humanos, 243, 103-135. https://dialnet.unirioja.es/servlet/articulo?codigo $=625060$

Bany-Ariffin, A. N., McGowan, C. B., Tunde, M. B., y Shahnaz, A. S. (2014). Top management characteristics and firm's international diversification activities: Evidence from a developing nation. Pertanika Journal of Social Science and Humanities, 22(December), 39-54. h https://bit.ly/3BV8S0X

Bhayana, C., Gupta, V., y Sharda, K. (2021). The Role of Shared Leadership in Managing Conflicts in Multigenerational Teams: A Research Framework. Business Perspectives and Research, 9(2), 252-268. https://doi.org/10.1177/2278533720964928

Camino-Mogro, S., y Brito-Gaona, L. F. (2021). Ciclicidad de la política fiscal en Ecuador. Revista de análisis económico, 36(1), 49-84. https://doi.org/10.4067/S0718-88702021000100049

Chen, J., Miller, D., y Chen, M.-J. (2021). Top management team time horizon blending and organizational ambidexterity. Strategic Organization, 19(2), 183-206. https://doi.org/10.1177/1476127019883669

Cortes, A. F., y Herrmann, P. (2021). Strategic Leadership of Innovation: A Framework for Future Research. International Journal of Management Reviews, 23(2), 224-243. https://doi.org/10.1111/ijmr.12246

Delgado, L. D., Sánchez, W. C., y Vélez, A. R. (2019). World-class managers: A pilot tuning project-based on generic competences. International Journal of Psychological Research, 12(2), 71-81. https://doi.org/10.21500/20112084.3941

Diéguez, M., Gueimonde, A., y Sinde, A. (2010). Éxito Empresarial Y Género En Turismo Rural. $\begin{array}{lllll}\text { Tourism } & y & \text { Management } & \text { Studies, } & 6,\end{array}$ https://dialnet.unirioja.es/servlet/articulo?codigo=3887980

Firk, S., Richter, S., y Wolff, M. (2021). Does value-based management facilitate managerial decision-making? An analysis of divestiture decisions. Management Accounting Research, 51(July 2019), 100736. https://doi.org/10.1016/j.mar.2021.100736

Flores-Romero, B., y González-Santoyo, F. (2019). Factores que inciden del desempeño de las MIPYMES en Michoacán, México: Un enfoque de la teoría de los efectos olvidados. Revista Mexicana de Economía y Finanzas, 14(1), 95-112. https://doi.org/10.21919/remef.v14i1.361

Gahan, P., Theilacker, M., Adamovic, M., Choi, D., Harley, B., Healy, J., y Olsen, J. E. (2021). Between fit and flexibility? The benefits of high-performance work practices and leadership capability for innovation outcomes. Human Resource Management Journal, 31(2), 414-437. https://doi.org/10.1111/1748-8583.12316

García, P., Cardona, P., y Chinchilla, M. N. (2001). Las competencias directivas mas valoradas. 
Occasional Paper - Universidad de Navarra, 3(01/04), 26. https://media.iese.edu/research/pdfs/OP-01-04.pdf

García, M., González, C. H., y Murillo, G. (2017). Características de las pymes según la gerencia sea hombre o mujer: caso empírico de Cali. Revista EAN, 82, 147-164. https://doi.org/10.21158/01208160.n82.2017.1648

Hoang, G., Wilson-Evered, E., y Lockstone-Binney, L. (2020). Leaders influencing innovation. Employee Relations: The International Journal, 43(2), 416-437. https://doi.org/10.1108/ER-07-2019-0279

Krén, H., y Séllei, B. (2020). The Role of Emotional Intelligence in Organizational Performance. Periodica Polytechnica Social and Management Sciences, 29(1), 1-9. https://doi.org/10.3311/PPso.15879

Lalic, B., Ciric, D., Gracanin, D., y Anisic, Z. (2019). The Importance of Education in Enhancing the Innovation Capacity in Serbia. En Springer Proceedings in Mathematics and Statistics (Vol. 281, pp. 63-71). Springer International Publishing. https://doi.org/10.1007/978-3030-14973-4_6

León, J. (2018). Relación entre tiempo de permanencia en el cargo de los directores y la calidad de gestión administrativa en las Instituciones Educativas de la RED 4 UGEL Ventanilla. En Universidad Nacional de Educación Enrique Guzmán y Valle (Vol. 1). https://repositorio.une.edu.pe/handle/UNE/2952

Li, P.-Y. (2017). The impact of the top management teams' knowledge and experience on strategic decisions and performance. Journal of Management y Organization, 23(4), 504-523. https://doi.org/10.1017/jmo.2016.24

Martínez, F. (2009). La formación universitaria versus las necesidades empresariales en el marco del EEES. La cuestión universitaria, 5, 181-191. http://polired.upm.es/index.php/lacuestionuniversitaria/article/view/3347/3411

McCormick, S. T., Kurth, N. K., Chambless, C. E., Ipsen, C., y Hall, J. P. (2021). Case Management Strategies to Promote Employment for Transition-Age Youth With Disabilities. Career Development and Transition for Exceptional Individuals, 44(2), 120131. https://doi.org/10.1177/2165143421991826

Men, L. R. (2012). CEO credibility, perceived organizational reputation, and employee engagement. Public Relations Review, 38(1), 171-173. https://doi.org/10.1016/j.pubrev.2011.12.011

Mishra, C. S. (2021). Does Managerial Ability Drive Firm Innovativeness? IEEE Transactions on Engineering Management, 68(4), 1139-1154. https://doi.org/10.1109/TEM.2019.2923892

Montiel, A. J. U. (2021). Economías de Aglomeración Y. Cuadernos de Economía, 40(82), 165191. https://doi.org/10.15446/cuad.econ.v40n82.81058

Nunes, M., y Pereira, R. (2021). Business Model Innovation And Business Performance In An Innovative Environment. International Journal of Innovation Management, 25(03), 2150036. https://doi.org/10.1142/S1363919621500365

Odiri, V., y Ideh, A. (2021). Determinants of Organizational Performance in Nigeria: Evidence from Service Firms. Humanities and Social Sciences Letters, 9(1), 86-95. https://doi.org/10.18488/journal.73.2021.91.86.95

Ogunfowora, B. (2014). The impact of ethical leadership within the recruitment context: The roles of organizational reputation, applicant personality, and value congruence. The Leadership Quarterly, 25(3), 528-543. https://doi.org/10.1016/j.leaqua.2013.11.013

Omar, A. y Pérez, C. (2014). Compromiso Organizacional, Género, Y Su Relacion Con Las 
Utilidades En Las Empresas. Revista electronica de ciencias sociales, 6(17), 1-17. https://www.redalyc.org/pdf/399/39915685007.pdf

Oorschot, J., Moscardo, G., y Blackman, A. (2021). Leadership style and psychological contract. Australian Journal of Career Development, 30(1), 43-54. https://doi.org/10.1177/1038416220983483

Ramírez, M., Aguilar, J., y Portal, M. (2018). The Impact of Economic and Financial Management Practices on the Performance of Mexican Micro-Enterprises: A Multivariate Analysis. Review of Business Management, 20(3), 319-337. https://doi.org/10.7819/rbgn.v20i3.3518

Ramos, A., Barberá, E., y Sarrió, M. (2003). Mujeres directivas, espacio de poder y relaciones de género. Anuario de Psicologia, 34(2), 267-278. https://revistes.ub.edu/index.php/Anuariopsicologia/article/view/8751

Rueda, J. (2011). La Profesionalización, Elemento Clave Del Éxito de la Empresa Familiar. Visión de Futuro - Universidad Nacional de Misiones, 15(1). https://www.redalyc.org/pdf/3579/357935477001.pdf

Saavedra, M. L. (2018). Diferencias en la competitividad de las empresas. INNOVA Research Journal, 3(10.1), 55-67. https://doi.org/10.33890/innova.v3.n10.1.2018.785

Sant, S. (2016). An Analysis of Credibility of CEO's in an Organisation Linkage with Employee Engagement. Asian Social Science, 12(10), 127. https://doi.org/10.5539/ass.v12n10p127

Superintendencia de Compañías Valores y Seguros del Ecuador. (2021). Superintendencia de Compañias, Valores y Seguros del Ecuador. https://www.supercias.gob.ec/portalscvs/

Susanto, A. B., Titisari, P., y Prajitiasari, E. D. (2021). Enhance smes performance through implementing quality strategic leadership, trust in leader, strategic planning. Quality Access to $\quad$ Success, 22(181), 26-29. https://www.proquest.com/openview/bb3444388dbd775b84061cd892adf2ff/1?cbl=10464 13ypq-origsite $=$ gscholar

Tu, P. A., Huong, D. T., y Triet, P. M. (2020). The moderating effects of managers' experience and gender on internationalization and firm performance of manufacturing enterprises in Turkey. Accounting, 6(7), 1209-1216. https://doi.org/10.5267/j.ac.2020.9.006

Usman, M., Ali, M., Ogbonnaya, C., y Babalola, M. T. (2021). Fueling the intrapreneurial spirit: A closer look at how spiritual leadership motivates employee intrapreneurial behaviors. $\begin{array}{llll}\text { Tourism Management, } & \text { 83(February 2020), } & 104227 .\end{array}$ https://doi.org/10.1016/j.tourman.2020.104227

Varela, A. M. (2020). Pobreza y desigualdad en Ecuador: modelo de microsimulación de beneficio fiscal. Cuadernos de Economía, 39(81), 823-856. https://doi.org/10.15446/cuad.econ.v39n81.76001 